\title{
Identifying the information and mental health service needs of children with cystic fibrosis
}

\author{
Hilary Power ${ }^{1}$, Amanda Oliver ${ }^{1}$, Shelby Shivak ${ }^{1}$, Heather Switzer ${ }^{2}$, Rebecca Genoe ${ }^{1}$, \\ Donald Sharpe $^{1}$, and Kristi Wright ${ }^{1}$ \\ ${ }^{1}$ University of Regina \\ ${ }^{2}$ Wascana Rehabilitation Centre
}

February 9, 2022

\begin{abstract}
Objective: Children with cystic fibrosis ( $\mathrm{CF}$ ) may experience elevated symptoms of depression and anxiety, as well as impairments in quality of life. To date, there is no mental health program specifically designed to address the mental health needs of children with CF. In the interest of informing the development of an accessible (i.e., Internet-delivered) mental health program, the present study examined the information and service needs of children with $\mathrm{CF}$ from the perspective of children with $\mathrm{CF}$, their parents, and CF health care providers. Methods: A qualitative research design was used. Participants $(\mathrm{n}=16)$ included children with $\mathrm{CF}(\mathrm{n}=5$, Mage $=9.25, \mathrm{SD}=1.29)$, parents $(\mathrm{n}=7$, Mage $=36.43, \mathrm{SD}=3.46)$, and health care providers $(\mathrm{n}$ $=4$, Mage $=44.00, \mathrm{SD}=10.46)$ recruited from regional $\mathrm{CF}$ clinics. Participants completed a brief demographic questionnaire. Semi-structured individual interviews were conducted with all participants. Results: Thematic content analysis generated four major themes: (1) challenges living with CF, (2) coping, (3) building independence, and (4) bridging gaps in services. Each theme was comprised of several subthemes. Conclusions: The findings highlight many emotional and social challenges experienced by children with $\mathrm{CF}$ and their families. Providing effective support for the entire family in managing and coping with $\mathrm{CF}$ was emphasized. Information gathered in the present study will be used, in combination with the empirical literature, to inform the development of an Internet-delivered mental health prevention program for children living with CF.
\end{abstract}

\section{IDENTIFYING THE INFORMATION AND MENTAL HEALTH SERVICE NEEDS}

\section{OF CHILDREN WITH CYSTIC FIBROSIS}

Hilary A. Power MA ${ }^{1}$, Amanda M. Oliver BA (Hons.) ${ }^{1}$, Shelby M. Shivak MA ${ }^{1}$, Heather Switzer $\mathrm{PhD}^{2}$, M. Rebecca Genoe $\mathrm{PhD}^{3}$, Donald Sharpe $\mathrm{PhD}^{1}$, \& Kristi D. Wright $\mathrm{PhD}^{1}$

${ }^{1}$ Department of Psychology, University of Regina, Regina, SK, Canada

${ }^{2}$ Wascana Rehabilitation Centre, Saskatchewan Health Authority, Regina, SK, Canada

${ }^{3}$ Faculty of Kinesiology and Health Studies, University of Regina, Regina, SK, Canada

This work was supported by the University of Regina, Saskatchewan Health Research Foundation, and the Saskatchewan Centre for Patient-Oriented Research. This paper was presented as a poster presentation at the North American Cystic Fibrosis Conference in Nashville, TN, USA in October 2019.

\section{Corresponding author:}

Hilary A. Power, M. A.

E-mail: hilaryp045@gmail.com 
Tel: $1-709-427-8530$

Postal address: University of Regina, Department of Psychology, 3737 Wascana Parkway, Regina, SK, Canada, S4S 0A2

Keywords: cystic fibrosis, children, mental health, qualitative methods, coping, treatment

Running head: MENTAL HEALTH SERVICE NEEDS OF CHILDREN WITH CF

Abstract

Objective: Children with CF may experience elevated symptoms of depression and anxiety, as well as impairments in quality of life. To date, there is no mental health program specifically designed to address the mental health needs of children with CF. In the interest of informing the development of an accessible (i.e., Internet-delivered) mental health program, the present study examined the information and service needs of children with $\mathrm{CF}$ from the perspective of children with $\mathrm{CF}$, their parents, and $\mathrm{CF}$ health care providers.

Methods: A qualitative research design was used. Participants $(n=16)$ included children with $\mathrm{CF}(n=$ $\left.5, M_{\text {age }}=9.25, S D=1.29\right)$, parents $\left(n=7, M_{\text {age }}=36.43, S D=3.46\right)$, and health care providers $(n=4$, $M_{\text {age }}=44.00, S D=10.46$ ) recruited from regional CF clinics. Participants completed a brief demographic questionnaire. Semi-structured individual interviews were conducted with all participants.

Results: Thematic content analysis generated four major themes: (1) challenges living with CF, (2) coping, (3) building independence, and (4) bridging gaps in services. Each theme was comprised of several subthemes.

Conclusions: The findings highlight many emotional and social challenges experienced by children with $\mathrm{CF}$ and their families. Providing effective support for the entire family in managing and coping with CF was emphasized. Information gathered in the present study will be used, in combination with the empirical literature, to inform the development of an Internet-delivered mental health prevention program for children living with CF.

Keywords: cystic fibrosis, children, mental health, qualitative methods, coping, treatment

\section{Introduction}

Children with Cystic Fibrosis (CF) may experience elevated psychological symptoms (e.g., depression, anxiety) ${ }^{1-3}$, as well as impairments in quality of life ${ }^{4}$. Psychological symptoms in children with CF pose a significant concern, as there are deleterious associations between psychological symptoms and health outcomes ${ }^{4-5}$. To address these mental health concerns and the barriers in traditional methods of mental health service delivery (e.g., limited resources, required travel), novel forms of service delivery (i.e., Internet-based programs) have been proposed as being viable and effective options ${ }^{6}$. Innovative technology-delivered treatments for children with CF have been developed in other areas of CF care (e.g., physiotherapy, nutrition) ${ }^{7-8}$. Despite Internet-delivered programs being developed in other areas of CF care and in psychological programs for family members impacted by $\mathrm{CF}^{6}$, to date there are no Internet-delivered mental health programs for children with CF. Prior to designing an Internet-delivered mental health program for children with CF, it is necessary to understand their information and service needs to tailor services appropriately ${ }^{9}$.

The purpose of the current study was to explore the information and mental health service needs of children with $\mathrm{CF}$ from the perspective of children with $\mathrm{CF}$, their parents, and health care providers (HCPs). There were five primary research questions: (1) What type of information about CF do children with CF need?; (2) What challenges do children with CF face?; (3) What types of services do children with CF need?; (4) What are the perceived benefits/drawbacks of obtaining service via the Internet?; and (5) What components should be included in an interactive, Internet-delivered mental health program for children with CF?

\section{Methods}

Study design 
A descriptive qualitative research design from a social-constructivist position was employed in the current study ${ }^{10}$. Qualitative research designs have been employed to inform the development of Internet-based mental health programs (e.g., capturing the experiences of those who will participate in the program, identifying program components, directing levels of program engagement $)^{11-12}$.

Participants

Three groups of participants were recruited for the study: (1) children with CF, (2) parents of children with CF, and (3) HCPs of children with CF. All participants were recruited from regional CF clinics. Child eligibility criteria included: (1) aged 7 to 18 years old; (2) CF diagnosis, and (3) spoke and read English. Child exclusion criteria included: (1) severe cognitive impairment and (2) major comorbid medical or psychiatric illness. Parents of children with $\mathrm{CF}$ were eligible if they spoke and read English. HCPs of children with CF were eligible if they were: (1) working with CF for a minimum of one year and (2) spoke and read English.

Written informed consent was obtained from adult participants. Parent caregivers provided written consent on behalf of their child and the children provided verbal assent. Ethics approval was provided by university and regional health authority research ethics boards.

Materials

Interview guides. The interview questions (see Table 1) were informed by the research objectives and the existing literature ${ }^{13}$, combined with the clinical experience of the research team. Follow-up questions were asked to allow participants to elaborate on their responses. Interviewing techniques (e.g., establishing rapport, active listening) were employed ${ }^{14}$.

Procedure

Individual semi-structured interviews were completed by the first author, either face-to-face or over the phone. When requested, parents were present during the child interviews. Individual interviews were also conducted with parents and HCPs of children with CF. Interviews ranged from 30 to 90 minutes in length. All interviews were audio-taped and field notes were made during the interviews. All participants were offered a $\$ 25$ cash honorarium for their participation.

\subsection{Analysis}

Demographic data were analyzed using IBM SPSS Statistics-Version 24 and summarized as means and standard deviations for continuous data and frequencies for categorical data. Interviews were audiotaped and transcribed verbatim. All transcripts were verified against the audio-recordings by the first author and imported into a qualitative analysis software program NVivo 12 .

Thematic content analysis was used to analyze the information gathered during semi-structured interviews 15. The six phases of thematic content analysis were conducted independently by two members (HP and $\mathrm{AO}$ ) of the research team to generate the main themes in participants' responses ${ }^{15}$. Disagreements in identification of themes were handled through consensus of the two analysts (HP and AO). As the main themes that emerged from each participant group overlapped across the entire sample and no theme was solely identified by only one participant group, the resulting themes were collapsed across all participants.

\section{Results}

Summary of Participants

Sixteen participants completed the study combined across three groups. Demographic characteristics for the child, parent, and HCP participants are summarized in Table 2.

Qualitative Results

Based on the overlapping perspectives across the three groups of participants, saturation was achieved 16-18. Four major themes emerged (with subthemes): (1) challenges living with CF; (2) coping; (3) building 
independence; and (4) bridging gaps in services. Samples of direct quotes are included in Table 3 to provide support for themes and subthemes.

Challenges to living with $\mathrm{CF}$

Emotional challenges

Common emotions experienced by children with CF included worry, fear, and confusion, often about medical procedures and the progression of the disease. As children learned more about the nature of living with a progressive disease, they experienced greater worries, and these worries had a functional impact (e.g., difficulty concentrating at school due to worrying about health status).

Social challenges

Social challenges experienced by children with CF included feeling different from peers. Children with CF must spend time completing various treatments that their peers do not and may miss out on activities. Participants also described children feeling self-conscious about $\mathrm{CF}$ and attempting to hide noticeable aspects (e.g., coughing) of their condition to avoid appearing different from others. Children with CF experienced feelings of worry, shame, or guilt, related to how their CF is impacting their families. As a result, children attempted to provide reassurance to their parents that they are doing well in attempts to reduce parental stress. Finally, participants believed children with CF had little connection with other children or adults with CF.

\section{Treatment challenges}

Self-managing treatment regimens were described as placing an extra responsibility on children with CF. Participants described the time-consuming nature of treatments and how treatments often interfered with other activities that children would rather spend their time doing. Moreover, parents expressed the difficulty their children had adhering to treatment regimes when their mood is low. Parents also highlighted the difficulty in balancing the enforcement of their child's treatment regimen, with allowing for flexibility in adherence in efforts to improve their child's mental well-being.

\subsubsection{Building independence}

\section{Understanding $\mathrm{CF}$}

All participants acknowledged that children need general disease knowledge about CF to build their independence in self-management and overall treatment adherence. Parents and HCPs highlighted the importance of children understanding the rationale for completing treatments. Parents explained that the lack of tangible reward for completing treatments makes it difficult for children to understand the importance of maintaining consistent treatment routines. Education surrounding the long-term benefits of completing treatments was highlighted as being important in motivating children to consistently self-manage their CF.

One challenging aspect of learning about CF reported was life expectancy.

Parents and HCPs differed in their opinions on how much information should be revealed to children about CF. Many parents described worrying about how their child would find out about sensitive issues related to having CF, such as disease prognosis. Parents emphasized the importance of delivering this information in a developmentally appropriate way, however they expressed uncertainty about what age is appropriate to begin having these discussions. HCPs described how some parents limit the amount of information they provide to children regarding life expectancy to protect them. While the latter was interpreted as a strategy employed to protect their children, HCPs perceived that censoring information is unhelpful in the long-term.

Mechanisms of learning

Participants described how children learn to manage CF. Parents were described as the primary source to help their children learn about CF and how to manage it. Parents' knowledge of treatments and openness to encouraging their child to self-manage their $\mathrm{CF}$ was discussed as being important in learning about $\mathrm{CF}$ 
and building independence. HCPs also contribute to CF learning and discussed directing conversations toward and obtaining information from children during CF clinics in order to allow children to advocate for themselves. Using this strategy encourages children to become more comfortable reporting their health status independently, and allows them to clarify their beliefs and feelings that may not be represented in their parents' reports.

Increasing responsibility

Increasing the ownership of the child in self-managing their CF over time was described by participants as critical. Parental beliefs were identified as a factor that could prevent children from learning to be independent in managing their own care. Parents and HCPs explained that it can be difficult for parents to let go of control of managing their child's treatments. Some parents indicated that it can be more efficient to complete a task for their child rather than watching them fail; other parents highlighted the importance of letting their child fail as a mechanism to facilitate independence. HCPs noted that having parents solely complete treatments for their child may be detrimental to their child's ability to self-manage their CF in the future. Child self-management of $\mathrm{CF}$ was believed to contribute to their overall self-confidence.

Some children felt that the abundance of disease information and the number of treatments required to complete when combined with other life activities (e.g., school, friendships) can be overwhelming. Attentional difficulties (e.g., Attention-Deficit Hyperactivity Disorder) were identified as a barrier to managing treatments independently (e.g., remembering to take medication). Parents highlighted the role of mental health in a child's ability to manage CF independently (e.g., treatment adherence was poorer when their child experienced low mood).

Coping

Focusing on positives

Participants described children coping with their CF by focusing on positive aspects of their lives. Understanding that they may be able to access effective treatments if their condition worsens was described as helpful in coping with the uncertainty of their disease progression. Other positive aspects identified included participating in fundraising initiatives and becoming a leader in the CF community.

Learning to cope from others

Participants reported that sharing with others and learning how to cope from other people is helpful. Children described talking with their parents about feelings associated with $\mathrm{CF}$ as a coping strategy. Parents highlighted the importance of creating an open environment and encouraging children to share how they are feeling. Parental modelling of helpful coping skills was discussed as being important to promote the use of helpful coping strategies in their children. HCPs emphasized the pivotal role that parents play in teaching their children about emotions and effective coping during difficult situations. Children added that it is helpful to know there are other people with $\mathrm{CF}$ who have similar experiences.

\section{Bridging gaps in services}

Services needed

Participants reported that the current major focus is the physical aspects of CF within care, while mental health care is not prioritized. Some HCPs described mental health care for children with CF as being reactive instead of preventative and how mental health services are not accessed until there is a crisis. Participants stressed that children with CF need opportunities to access mental health care consistently. Additionally, participants highlighted the importance of offering mental health services to other family members, such as parents and siblings of children with $\mathrm{CF}$.

\section{Barriers to access}

Barriers to accessing mental health services included financial barriers, the time-consuming nature of attending appointments, long waitlists, and travel to access services for families living in rural areas. HCPs 
added that parental beliefs about stigma associated with receiving mental services may prevent children from accessing the services that they need. Education surrounding mental health care and normalizing the experience of seeking out mental services was believed to be important in eliminating stigma as a barrier for accessing services.

Informing a novel form of service delivery

All participants expressed interest in an Internet-delivered mental health prevention program for children with CF. Participants emphasized the importance of children learning appropriate coping skills early to promote well-being. Potential drawbacks to this type of service delivery included safety concerns regarding Internet use and access to the Internet. Program usage or uptake could be impacted by time commitment required and HCP monitoring.

Finally, participants suggested components to be included in an Internet-delivered mental health program include education about $\mathrm{CF}$ and $\mathrm{CF}$ treatment, physical and mental health, emotions experienced by children with CF, coping strategies, how to access mental health services, and social support provision. Interactive aspects (e.g., interactive vignettes) and short modules to accommodate attention difficulties were also recommended.

\section{Discussion}

This is the first study to qualitatively investigate the challenges with and information and mental health service needs of children with CF from varying perspectives. The four themes and their associated subthemes provide us with critical insights and knowledge to address their mental health service needs. Children with CF experience a variety challenges associated with their condition (e,g., worries about health, disease outcomes, hospital procedures), challenges consistent with previous literature ${ }^{19-20}$. Social challenges based on their differing expectations and responsibilities compared to their healthy peers were also highlighted ${ }^{21}$. Connecting with other children and adolescents with CF was emphasized as an important way to normalize and validate these experiences. Including ways to cope with the uncertainty of the future, as well as providing social support through shared stories of peers with CF is critical when considering the development of mental health programs for this population.

The importance of children with CF learning about their CF and how to manage it independently was prominent in our results. Previous literature has similarly demonstrated that appropriate education can help facilitate effective management and coping with $\mathrm{CF}$, promoting treatment adherence and overall wellbeing ${ }^{22-23}$. An important step to relieve anxiety and improve disease management may be through providing CF-related education to children with CF. Opinions concerning disclosing information about life expectancy and disease prognosis to children differed between parent and HCP participants. Limited research has focused on how and when individuals with CF should receive information about their prognosis ${ }^{23-24}$. One study demonstrated that patients desire early, individualized communication about prognosis and that ageappropriate educational materials could aid in initiating these conversations among families and $\mathrm{HCPs}^{25}$. Deciding what information about disease prognosis related to CF should be presented and how to present that information are important considerations when considering the development of an Internet-delivered mental health program for children with CF.

Results outlined the ways children with CF cope with their illness. Strategies included focusing on positive aspects of their life and remaining hopeful in their ability to access effective treatments. These strategies are consistent with previous research that has emphasized the importance of optimism and acceptance in coping with $\mathrm{CF}^{25}$. Strategies to promote positive coping were highlighted as well (e.g., discussions with parents about emotions associated with CF; parental modelling of helpful coping skills). Moreover, child participants noted that it is helpful to know there are other people with CF that have shared experiences. Research across various chronic illness populations have demonstrated that greater levels of social support from friends and family, or others with an ability to understand one's experience, has been associated with greater adherence to treatments and better quality of life ${ }^{26-27}$. Emotional support from friends and family, as well interactions with other youth with $\mathrm{CF}$, has been recommended to promote feelings of connectedness 
and positive coping ${ }^{21}$.

Directions for mental health care services for children with CF were discussed. Preventative mental health care was recommended to prepare children to cope effectively with potential challenges they may face in the future. Participants offered specific feedback about the development of an Internet-delivered mental health program for children with CF. Developing and implementing a flexible, stepped-care approach to care where children with $\mathrm{CF}$ would receive a mental health prevention program followed by further intervention for children with more acute needs may be beneficial.

The current study offers valuable insights into the challenges of children with $\mathrm{CF}$ and their families, as well as the information and services they need, and their views on receiving and Internet-delivered mental health program. A primary strength of the current study is the inclusion of three different perspectives (i.e., children with CF, parents, and HCPs). By including these different perspectives, a more complete understanding of the experiences of children with CF was achieved. Several studies have excluded children under the age of 12 years. By including younger aged children in the present study allowed the experiences of this age group to be represented.

There are limitations to consider. First, as the study included the participation of young children, building rapport was essential prior to interviews to ensure children felt comfortable sharing information about their experiences living with CF. As the time to build rapport was often short, children may not have shared in-depth details of their experiences. Additionally, based on their age and developmental understanding of their condition, children may not have possessed further information or insights to share. Second, all of the participants were residents of one province in Canada, and their experiences, particularly related to the health care system and availability of services within CF clinics, may not generalize to other provinces within Canada or beyond.

\section{Conclusions}

The results of the current study will direct the development of an Internet-delivered mental health prevention program for children with CF. In particular, our findings will be used to tailor program content to the needs of the individuals for whom the program is designed. It is anticipated that the developed program will aid in the prevention of mental health concerns (e.g., depressed affect, anxiety) in children with CF.

\section{Acknowledgements}

We would like to thank the families and health care providers who participated in the study.

\section{Funding}

This work was supported by the University of Regina, Saskatchewan Health Research Foundation, and the Saskatchewan Centre for Patient-Oriented Research.

\section{Conflict of Interest Statement}

All authors report no conflicts of interest.

\section{References}

1. Gundogu U, Fis NP, Eralp EE, Karadag BT. Major depression and psychiatric comorbidity in Turkish children and adolescents with cystic fibrosis. Pediatr Pulmonol 2019;54(12): 1927-1935.

2. Quittner AL, Goldbeck L, Abbott J, Duff A, Lambrecht P, Solé A, Tibosch MM, Bergsten Brucefors A, Yukshel H, Catastini P, et al. Prevalence of depression and anxiety in patients with cystic fibrosis and parent caregivers: Results of The International Depression Epidemiological Study across nine countries. Thorax 2014;69(12):1090-1097.

3. Reay M, Puckey M. Psychological well-being of cystic fibrosis patients ages 4-10 years. J Cyst Fibros 2019;8 (1):S184.

4. Riekert KA, Bartlett SJ, Boyle MP, Krishnan JA, Rand CS. The association between depression, lung function, and health-related quality of life among adults with cystic fibrosis. Chest 2007;132(1):231-237. 
5. Cox NS, Wilson CJ, Bennett KA, Johnston K, Potter A, Chang AB, Lee AL. Health-related quality of life and psychological well-being are poor in children with bronchiectasis and their parents. ERJ Open Res 2019;5(3):00063.

6. Fidika A, Herle M, Lehmann C, Weiss C, Knaevelsrud C, Goldbeck L. A web-based psychological support program for caregivers of children with cystic fibrosis: A pilot study. Health Qual Life Outcomes 2015;13(11):1-9.

7. Calvo-Lerma J, Martinez-Jimenez CP, Lazaro-Ramos J, Andres A, Crespo-Escobar P, Stav E, Schauber C, Pannese L, Hulst JM, Suarez L, et al. Innovative approach for self-management and social welfare of children with cystic fibrosis in Europe: Development, validation, and implementation of an mHealth tool (MyCyFAPP). Br Med J 2017;7(3):e014931.

8. Lang RL, Wilson C, Stockton K, Russell T, Johnston LM. CyFit telehealth: Protocol for a randomised controlled trial of an outpatient physiotherapy service for children with cystic fibrosis. BMC Pulm Med 2019;19 (1):21.

9. Everhart RS, Molitor SJ, Wentz D, Schmidt HD, Schechter MS. Identifying and integrating parent priorities for psychosocial support services in a pediatric cystic fibrosis clinic. J Clin Psychol Med Settings 2019;26(2):235-241.

10. Sandelowski M. Whatever happened to qualitative description? Res Nurs Health 2000;23:334-340.

11. Chan JKY, Farrer LM, Gulliver A, Bennett K, Griffiths KM. University students' views on the perceived benefits and drawbacks of seeking help for mental health problems on the Internet: A qualitative study. JMIR Hum Factors 2016;3:e3.

12. Garmy P, Berg A, Clausson E. A qualitative study exploring adolescents' experiences with a schoolbased mental health program. BMC Public Health 2015;15:1074.

13. Stinson J, Lalloo C, Harris L, Cafazzo J, Campell F, Chorney J, Clark J, Dick B, Forgeon P, et al. iCanCope with Pain: User-centered design of an integrated smartphone and web-based pain selfmanagement program for youth and young adults with chronic pain. J Pain 2016;17(4):S109.

14. Morgan DL. 1997. Focus groups as qualitative research (2 ${ }^{\text {nd }}$ ed.). Sage Publications.

15. Braun KL, Clarke V. Using thematic analysis in psychology. Qual Res Psychol 2006;3:77-101.

16. Guest G, Bunce A, Johnson L. How many interviews are enough? An experiment with data saturation and variability. Field Methods 2006;18:59-82.

17. Saunders B, Sim J, Kingstone T, Baker S, Waterfield J, Bartlam B, Burroughs H, Jinks C. Saturation in qualitative research: Exploring its conceptualization and operationalization. Qual Quant 2017;52(4):115.

18. Urquhart C. 2013. Grounded theory for qualitative research: A practical guide. Sage.

19. Buhr K, Dugas MJ. The role of fear of anxiety and intolerance of uncertainty in worry: An experimental manipulation. Behav Res Ther 2009;47(3):215-223.

20. Mitsmansgruber H, Smrekar U, Rabanser B, Beck T, Eder J, Ellemunter H. Psychological resilience and intolerance of uncertainty in coping with cystic fibrosis. J Cyst Fibros 2016;15(5):689-695.

21. Jamieson N, Fitzgerald D, Singh-Grewal D, Hanson CS, Craig JC, Tong A. Children's experiences of cystic fibrosis: A systematic review of qualitative studies. Pediatrics 2014;133(6):e1683-e1687.

22. Bregnballe V, Thastum M, Shiotz PO. Psychosocial problems in children with cystic fibrosis. Acta Paediatr 2007;96(1):58-61.

23. Dempster NR, Wildman BG, Masterson TL, Omlor GJ. Understanding treatment adherence with the Health Belief Model in children with cystic fibrosis. Health Educ Behav 2018;45(3):435-443.

24. Lonabaugh KP, O'Neal KS, McIntosh H, Condren M. Cystic fibrosis-related education: Are we meeting patient and caregiver expectations? Patient Educ Couns 2018;101(10):1865-1870.

25. Abbott J, Dodd M, Gee L, Webb, K. Ways of coping with cystic fibrosis: Implications for treatment adherence. Disabil Rehabil 2001;23(8):315-324.

26. Flewelling KD, Sellers DE, Sawicki GS, Robinson WM, Dill EJ. Social support is associated with fewer reported symptoms and decreased treatment burden in adults with cystic fibrosis. J Cyst Fibros 2019;18(4):572-576.

27. Helms SW, Dellon EP, Prinstein MJ. Friendship quality and health-related outcomes among adolescents 
with cystic fibrosis. J Pediatr Psychol 2015;40(3):349-358.

\section{Hosted file}

Power et al CF qualitative manu tables Ped Pulm.docx available at https://authorea.com/users/ 459732/articles/555902-identifying-the-information-and-mental-health-service-needs-ofchildren-with-cystic-fibrosis 